\title{
Research on the Correlation of Financial Risk based on Copula Technology
}

\author{
Yafei Chen, Huanlu Yang, Xuan Shang \\ College of Economics and Management, Zhejiang University of Technology \\ cyf568331@126.com
}

\begin{abstract}
Based on the Copula function, we study the effectiveness of the method in dealing with the nonlinear related structure between the financial risks .we run an empirical analysis according to the data of daily return rate of SSE Composite Index and Shenzhen Composite Index for the ten years of 2007-2017. By using the Kernel-distribution estimation method, the distribution of the sample data was determined, then the parameters of the five selected models were estimated and the correlation coefficients were calculated. Finally, the Square Euclidean was calculated to determine the optimal function model. The results support that Copula function, has become a more effective method of dealing with the relevant structure between variables owing to its excellent features, in which the $t$-Copula function can describe the related structure of financial assets more accurately, which plays an important guiding role in the prediction and management of financial risk.
\end{abstract}

Keywords-Copula function; Financial risk; Correlation; Risk management

\section{INTRODUCTION}

The financial market is a relatively large and complex system. There is a certain relationship between the markets and between the risk factors. The correlation analysis of the financial risks has always been a relatively cutting-edge problem. The study of the characteristics of the financial risk and the related structure between the risks plays an important role in risk analysis and risk management. Previous studies on the correlation of financial risks are mostly based on linear correlation and normality. However, with the rapid development of the financial market, the relationship between financial risks is more complex, there are more non-linear, asymmetrical relations showed between financial data, instead of simple linear relationship, and the data have the characteristics of peak, thick tail and time varying. The original research methods have not effectively studied the complex correlation among financial markets due to its limitations [1].

Under the background described above, the technology and methods to study the correlation of financial risks are also developing. The Copula function, with its many excellent properties, becomes more effective in dealing with the complex relationship between variables. It can be widely used in time series analysis, insurance pricing, risk analysis and so on [2-3]. There are many other good statistical properties of the Copula function, for example, the measure of consistency and relevance obtained by using this method keeps unchanged under the strictly monotonic transformation. Furthermore, in financial data analysis, the function can also connect the marginal distribution and the joint distribution of the random variables, and then accurately express the correlation structure between the variables [4]. Therefore, it is a very effective choice to apply the Copula function to the study of practical problems in the financial field.

\section{COPULA FUNCTION THEORY AND CORRELATION MEASUREMENT}

The Copula function is a statistical theory method that can effectively capture the asymmetrical and nonlinear relations between variables. the definition of the Copula function was first proposed by Sklar in 1959. And In 1999, the following description was made by Nelsen [5]:

A function $C(\cdot)$ defined by $I^{2}=[0,1]^{2} \rightarrow \mathrm{I}$, which can satisfy the following properties [6], is called the binary copula function:

(1) The function $\mathrm{C}(\cdot)$ is monotonous and non decreasing for every variable;

(2) For any $\mathrm{u}, \mathrm{v} \in[0,1]$, there are $\mathrm{C}(u, 0)=C(0, v)=$ $0, \mathrm{C}(u, 1)=u$ and $\mathrm{C}(1, \mathrm{v})=\mathrm{v}$;

(3) For any $0 \leq u_{1} \leq u_{2} \leq 1$ and $0 \leq v_{1} \leq v_{2} \leq 1$, there are:

$$
\mathrm{C}\left(u_{2}, v_{2}\right)-C\left(u_{2}, v_{1}\right)-C\left(u_{1}, v_{2}\right)+C\left(u_{1}, v_{1}\right) \geq 0
$$

Commonly, there are two types of copula functions [7], one is the Elliptic Copula function, and the other is the Archimidean Copula function. The elliptic Copula function mainly includes the Gaussian Copula and t-Copula, in which the t-Copula function is more sensitive in the tail observation, and is more effective in dealing with the "peak thick tail" problem and the related structure between random variables of the financial data. There are three types of common Archimidean Copula functions: Clayton Copula function, Gumbel Copula function and Frank Copula function.

In addition to the function of connecting the marginal distribution function and the joint distribution function, the features of the Copula function does not change after strict monotone transformation of the variable is performed, and the correlation measurement index based on the Copula function will not change, therefore, this characteristic also has a more extensive application, frequently-used correlation measurement indices based on the Copula function include Kendall rank correlation coefficient, Spearman rank correlation coefficient and tail correlation coefficient. 


\section{EMPIRICAL ANALYSIS}

\section{A. Sample Selection and Statistical Description of Data}

This paper uses the data of the daily return of Shanghai Composite Index and Shenzhen composite index as the research object, the sample collection ranges from January 5, 2007 to January 5, 2017, with a total of 2434 groups of effective data, the rate of return $r_{t}$ is defined as $r_{t}=$ $\ln \left(P_{t}\right)-\ln \left(P_{t-1}\right), P_{t}$ is the closing price of stock on trading day $\mathrm{t}$, data origins from wind info, data analysis and processing is accomplished by Stata12.0 and Eviews8.0.

The chart of the trend of the closing price of Shanghai Composite Index and Shenzhen composite index is shown in Fig 1. S1 indicates the daily closing price series of Shanghai Composite Index, and S2 indicates the daily closing price series of Shenzhen composite index. Figures 2 and 3 separately give the return rate changes over time.

Fig 1 indicates that the trends of the daily closing price of the Shanghai Composite Index and the Shenzhen composite index are basically the same, indicating that there exists a certain interactivity and relevance between Shanghai stock market and Shenzhen stock market. Figures 2 and 3 give the basic statistical characteristics of the two return rate sequences.

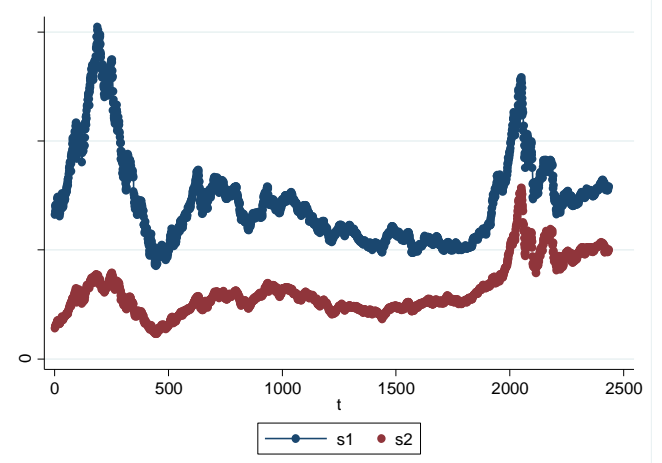

Fig. 1 Daily closing prices of Shanghai Composite Index and Shenzhen Composite Index

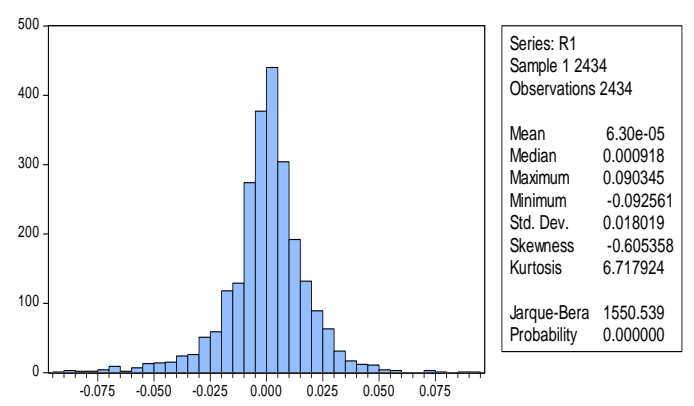

Fig. 2 The statistical description of the return rate sequence of Shanghai Composite Index

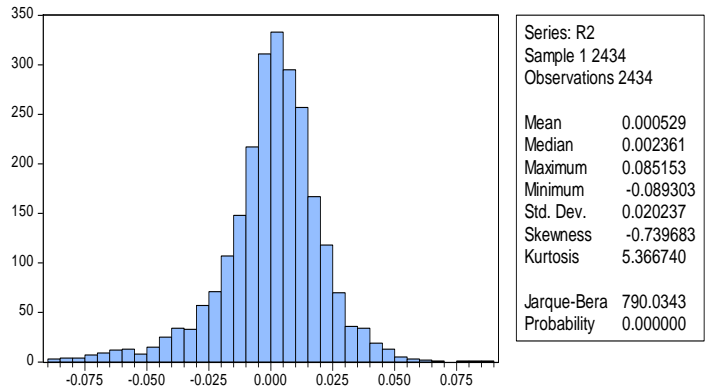

Fig. 3 The statistical description of the return rate sequence of Shenzhen Composite Index

We can see that the distribution of the daily return rate series of the two time series of Shanghai Composite Index and Shenzhen Composite index is basically symmetrical. The skewness of the two return rate sequence are negative, showing left partial distribution, and the kurtosis is more than 3 , two return rate sequence shows the feature of "peak and thick tail". The J-B statistics of Shanghai Composite index is 1550.539 , the J-B statistics of Shenzhen Composite index is 790.0343 , and the $\mathrm{P}$ value is 0 , which indicates that the two return rate sequences both reject the null hypothesis of obeying normal distribution.

\section{B. Determining the Marginal Distribution Using Nonparametric Method}

From the analysis above, we can see that the daily return rate sequences of Shanghai Composite Index and Shenzhen composite index do not obey the Gaussian distribution. It is difficult to find this type of distribution function from common distributions. Therefore, the distribution of the two return sequences should be determined by the non parametric method, and the following data processing is completed using MATLAB (R2012a). The kernel distribution estimation method [8] is used in the fitting of two return rate distributions, and the results are shown in Fig 4 and Fig 5.

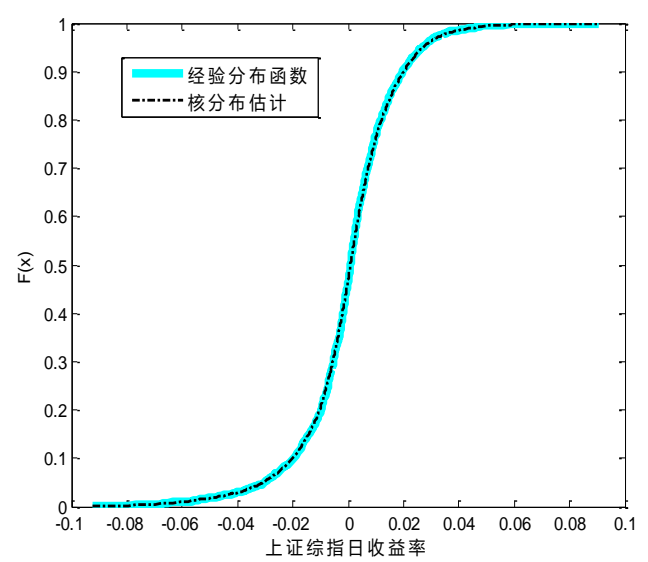

Fig. 4 Empirical distribution function and core distribution estimation of Shanghai Composite Index Returns 


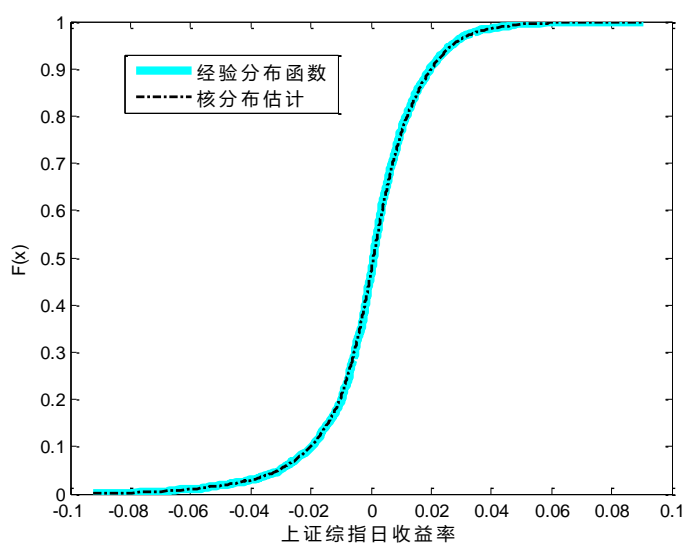

Fig. 5 Empirical distribution function and core distribution estimation of Shanghai Composite Index Returns

It can be seen from the figures above that the distribution of the return rate fitted by the nonparametric kernel distribution estimation is basically consistent with their respective empirical distribution functions, that is, the nonparametric kernel distribution estimation can better estimate the distribution of the sample population, which can be used as the marginal distribution function of the return of Shanghai Composite Index and Shenzhen Composite Index.

\section{The Selection of Optimal Copula Function}

\section{1) Drawing Binary Frequency Histogram}

The binary frequency histogram is drawn according to the marginal distribution of the two return rate variables above as an estimation of the joint density function of the two variables.
The binary frequency histogram of the two return rate sequences is shown in Fig 6.

From the binary frequency histogram, we can see that the joint density function of the two return rate sequences, that is, the Copula density function has the characteristic of asymmetric thick tail, then we use five different Copula functions to fit the related structure of the original data. Finally, we select the optimal Copula function according to the minimum principle of square Euclidean distance.

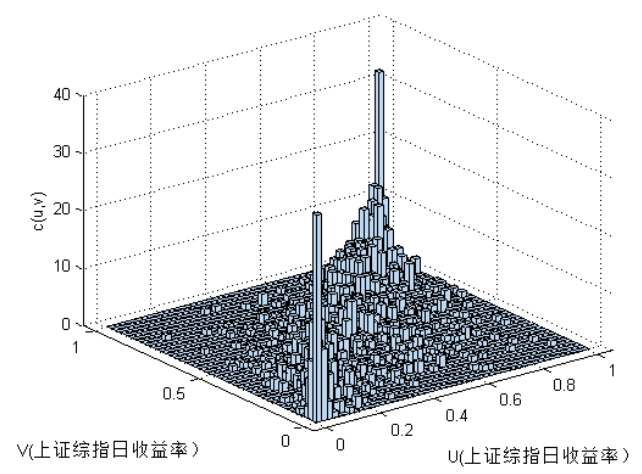

Fig. 6 The binary frequency histogram of the marginal distribution of Shanghai Composite Index and Shenzhen Composite Index daily rate of return

\section{2) Parameter Estimation}

In Matlab, we use the copula fit function to estimate the unknown parameters of different types of Copula functions. The results are shown in Table 1.

TABLE I RESULTS OF PARAMETER ESTIMATION OF DIFFERENT TYPES OF COPULA FUNCTION

\begin{tabular}{lc}
\hline Type of function & Parameter estimation \\
\hline Gaussian Copula function & $\hat{\rho}=\left(\begin{array}{lr}1.0000 & 0.8927 \\
0.8927 & 1.0000\end{array}\right)$ \\
t-Copula function & $\hat{\rho}=\left(\begin{array}{rr}1.0000 & 0.8995 \\
0.8995 & 1.0000\end{array}\right), \hat{k}=2.9083$ \\
Clayton-Copula function & $\hat{\alpha}=3.5467$ \\
Gumbel-Copula function & $\hat{\alpha}=3.3226$ \\
Frank-Copula function & $\hat{\alpha}=11.9957$ \\
\hline
\end{tabular}

3) Estimation of Rank Correlation Coefficient

Now that the parameter values of various Copula functions are obtained, the copulastat function is used to find the parameter estimates of various Copula functions, the Kendall rank correlation coefficient and the Spearman rank correlation coefficient of the original data. The results are shown in table 2.

By making a comparison between the Kendall rank correlation coefficient, the Spearman rank correlation coefficient of different Copula functions and the Kendall rank correlation coefficient, the Spearman rank correlation coefficient of the original data of return rate, it is seen that the linear correlation parameter $\hat{\rho}=0.8995, \hat{k}=2.9083$ is the degree of freedom of t-Copula function, this function reflects the Kendall correlation between Shanghai Composite Index and Shenzhen Composite Index daily rate of return data better, however, the Gumbel-Copula function with parameter $\hat{\alpha}=$ 3.3226 is better reflected the Spearman rank correlation between the daily data of the return rate of Shanghai Composite Index and Shenzhen Composite Index. 
TABLE II RANK CORRELATION COEFFICIENTS OF DIFFERENT TYPES OF COPULA FUNCTIONS

\begin{tabular}{llllll}
\hline Different types of Copula function & $\begin{array}{l}\text { Kendall } \\
\text { coefficient } \tau\end{array}$ & rank & correlation & $\begin{array}{l}\text { Spearman } \\
\text { coefficient } \rho_{s}\end{array}$ & rank correlation
\end{tabular}

\begin{tabular}{lll}
\hline Original data & 0.7076 & 0.8725 \\
Gaussian Copula function & 0.7023 & 0.8836 \\
t-Copula function & 0.7121 & 0.8909 \\
Clayton-Copula function & 0.6394 & 0.8233 \\
Gumbel-Copula function & 0.6990 & 0.8752 \\
& & \\
Frank-Copula function & 0.7123 & 0.8962
\end{tabular}

4) Calculating the Euclidean Square Distance of Different Copula Functions

According to the daily return rate data of Shanghai Composite Index and Shenzhen composite index, the empirical Copula function is introduced and the value of the empirical Copula function is obtained. Then the square Euclidean distance between the different Copula functions and the empirical Copula functions is calculated to measure the goodness of fit. The smaller the square Euclidean distance is, the better the fitting effect of the Copula function is. The results are shown in Table 3.

According to the square Euclidean distance principle, we can see that $\mathrm{t}$-Copula function has the highest goodness of fit in five types of Copula models, followed by Gaussian Copula

TABLE III THE SQUARE EUCLIDEAN DISTANCE BETWEEN VARIOUS COPULA FUNCTIONS AND THE EMPIRICAL COPULA FUNCTION

\begin{tabular}{cc}
\hline Type of function & square Euclidean distance \\
\hline Gaussian Copula function & 0.0448 \\
t-Copula function & 0.0257 \\
Clayton-Copula function & 0.8526 \\
Gumbel-Copula function & 0.1925 \\
Frank-Copula function & 0.2705
\end{tabular}

function, Gumbel-Copula function, Frank-Copula function and Clayton-Copula function. So the t-Copula function with the linear correlation parameter $\hat{\rho}=\left(\begin{array}{ll}1.0000 & 0.8995 \\ 0.8995 & 1.0000\end{array}\right)$ and the degree of freedom $\hat{k}=2.9083$ is optimal when fitting the correlation between Shanghai Composite Index and Shenzhen composite index's daily return rate variables, it can be used as a joint distribution function to measure the integration risk of Shanghai Composite Index and Shenzhen composite index.

Taking the parameter estimated above into the expression of t-Copula function, we can obtain the optimal Copula function that has been selected by our empirical study as below:

$$
\begin{aligned}
C(u, v ; 0.8995,3) & =\frac{1}{2 \pi \sqrt{1-0.8995^{2}}} \int_{-\infty}^{t_{3}^{-1}(u)} \int_{-\infty}^{t_{3}{ }^{-1}(v)}[1 \\
& \left.+\frac{x^{2}-2 \times 0.8995 x y+y^{2}}{3 \times\left(1-0.8995^{2}\right)}\right]^{-\frac{5}{2}} d x d y
\end{aligned}
$$

The density function diagram of the binary t-Copula function is given below:

From the density function graph of the binary t-Copula function of Fig 7, it can be seen that the density function of the $\mathrm{t}$-Copula function presents a more significant thick tail feature, which can more truly reflect the related structure between the two return rate variables. 


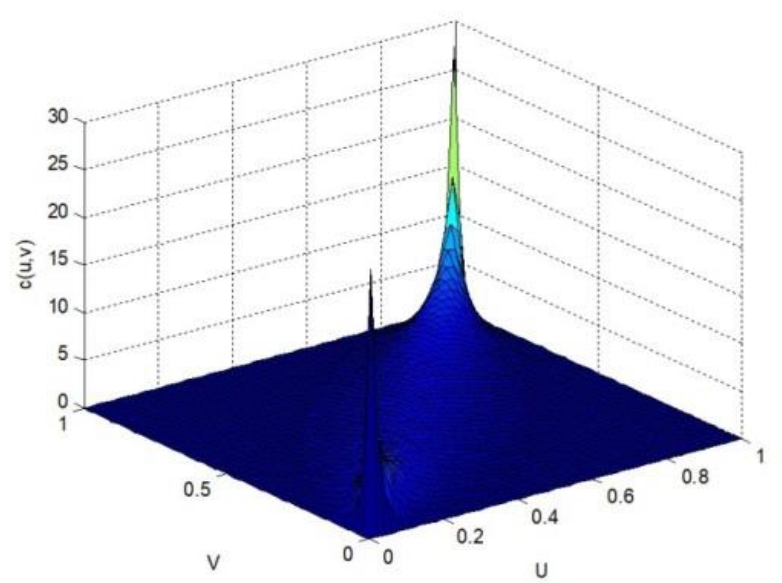

Fig. 7 The density function diagram of the binary t-Copula function

\section{CONCLUSIONS AND SUGGESTIONS}

This paper uses Copula technology to study the relationship between the daily return rate sequence of Shanghai Composite Index and Shenzhen composite index. The empirical results show that the binary t-Copula function is optimal when fitting the related structure between the two return rate variables, and it can also reflect the characteristics of the thick tail of financial data. Through comparison and analysis, it is known that t-Copula function is more accurate when fitting the related structure of variables, especially when dealing with the "peak and thick tail" problem of financial data. At the same time, risk managers can analyze the risk of financial markets by studying the related structure of the financial variables, especially the characteristics of the tail, and then carry out effective risk measurement and supervision. The investors can also forecast the change of the future stock market through studying the related structure of the variables, in order to avoid risks and make reasonable investments.

\section{REFERENCES}

[1] Longin F. Extreme Correlation of International Equity Markets[J]. Journal of Finance, 2001, 56(2):649-676.

[2] Li D X. On Default Correlation[J]. Journal of Fixed Income, 2009, 9(4):43-54.

[3] Bouyé E, Durrleman V, Nikeghbali A, et al. Copulas for Finance - A Reading Guide and Some Applications[J]. Social Science Electronic Publishing, 2007.

[4] Li D X. On Default Correlation: A Copula Function Approach[J]. Social Science Electronic Publishing, 1999, 9(4).

[5] Nelsen R B. An Introduction to Copulas[M]// An introduction to copulas. Springer, 2006:xx,315.

[6] Bouyé E, Durrleman V, Nikeghbali A, et al. Copulas for Finance - A Reading Guide and Some Applications[J]. Social Science Electronic Publishing, 2007.

[7] Sklar M. Fonctions de repartition a n dimensions et leurs marges[J]. Publ.inst.statist.univ.paris, 1959, 8.

[8] Hartmann P, Straetmans S, Vries C G D. Asset Market Linkages in Crisis Periods [J]. Review of Economics \& Statistics, 2004, 86(1):313-326. 